\title{
Keeping It Together: Mechanisms of Intersegmental Coordination for a Flexible Locomotor Behavior
}

\author{
Joshua G. Puhl and Karen A. Mesce \\ Graduate Program in Neuroscience and Departments of Entomology and Neuroscience, University of Minnesota, Saint Paul, Minnesota 55108
}

The coordination of multiple neural oscillators is key for the generation of productive locomotor movements. In the medicinal leech, we determined that activation and coordination of the segmental crawl oscillators, or unit burst generators, are dependent on signals descending from the cephalic ganglion. In nearly intact animals, removing descending input (reversibly with a sucrose block) prevented overt crawling, but not swimming. Cephalic depolarization was sufficient for coordination. To determine whether descending signals were necessary for the generation and maintenance of posterior-directed intersegmental phase delays, we induced fictive crawling in isolated whole nerve cords using dopamine (DA) and blocked descending inputs. After blockade, we observed a significant loss of intersegmental coordination. Appropriate phase delays were also absent in DA-treated chains of ganglia. In chains, when one ganglion was removed from its neighbors, crawling in that ganglion emerged robust and stable, underscoring that these oscillators operate best with either all or none of their intersegmental inputs. To study local oscillator coupling, we induced fictive crawling (with DA) in a single oscillator within a chain. Although appropriate intersegmental phase delays were always absent, when one ganglion was treated with DA, neighboring ganglia began to show crawl-like bursting, with motoneuron spikes/burst greatest in untreated posterior ganglia. We further determined that this local excitatory drive excluded the swim-gating cell, 204. In conclusion, both long-distance descending and local interoscillator coupling contribute to crawling. This dual contribution helps to explain the inherent flexibility of crawling, and provides a foundation for understanding other dynamic locomotor behaviors across animal groups.

\section{Introduction}

Animal locomotion occurs in many forms and encompasses a wide variety of rhythmic movements. Yet, across this array of activities, it is the coordination of multiple neural oscillators that is needed to generate fluid and productive locomotor behavior (Grillner, 2003). A core feature of this coordination is the so called "coupling" of central pattern generators (CPGs) within the CNS, which can employ one or more key strategies: (1) coupling that is inherent to the rhythm-generating circuitry, leading to greater stereotypy in the phase relationships between oscillators; and (2) coupling that is extrinsic to the unit oscillators. This latter strategy is often associated with a greater flexibility in behavioral output, but at the expense of the predictability provided by the first one. Because stereotypy is a beneficial attribute that renders a behavior more amenable to a physiological analysis, many studies of locomotion have revealed neural operations that are comprised of relatively tightly coupled oscillators. For example, experimental and modeling studies of swimming in the lamprey and leech, insect flight, and swimmeret beating in the crayfish have provided much of the foundation underlying what is cur-

Received Nov. 20, 2009; revised Dec. 30, 2009; accepted Jan. 5, 2010.

This work was supported by grants from the National Science Foundation (I0S-0523959 and 0924155 to K.A.M.) and the National Institutes of Health (individual National Research Service Award F31NS060332 to J.G.P). We are grateful to Kathleen Klukas for her expert technical assistance and preparation of illustrations. We also thank Roger Moon for advice on data analyses and William B. Kristan for his always enlightening discussions.

Correspondence should be addressed to Dr. Karen A. Mesce, Departments of Entomology and Neuroscience, University of Minnesota, 219 Hodson Hall, 1980 Folwell Avenue, St. Paul, MN 55108. E-mail: mesce001@umn.edu. DOI:10.1523/JNEUROSCI.5765-09.2010

Copyright $\odot 2010$ the authors $\quad 0270-6474 / 10 / 302373-11 \$ 15.00 / 0$ rently understood about the coordination of oscillators whose coupling is an emergent property of the oscillators themselves (Ikeda and Wiersma, 1964; Cohen and Wallén, 1980; Robertson and Pearson, 1983; Cang and Friesen, 2002; Smarandache et al., 2009). In contrast, other locomotor behaviors exhibiting much more variability are not as well understood at the cellular level (Kiehn and Butt, 2003).

Studies of walking in insects (Büschges et al., 2008), cats (Büschges, 2005), and humans (Yang and Gorassini, 2006), and those of crawling in the medicinal leech (Cacciatore et al., 2000), suggest that these locomotor events may employ oscillatorcoupling strategies to maximize flexibility. Some research has indicated that higher-order descending information may contribute to oscillator coupling for both leech crawling (Cacciatore et al., 2000; Cornford et al., 2006) and cat walking (Shimamura and Kogure, 1983; Drew et al., 1986), although more direct evidence is warranted. In the cat and human, investigations of oscillator coupling and descending control have been limited by the inherent complexities of the mammalian CNS and the locomotor behaviors themselves. Thus, to understand better how a relatively slow and variable locomotor behavior is controlled, we turned to a study of crawling behavior in the medicinal leech.

Briefly, leech crawling consists of alternating elongation and contractions of the whole body with coordinated attachment and release of the anterior and posterior suckers. Segmental movements always progress along the body in an anterior to posterior direction, but they are often dynamic and can be suspended or modulated during each elongation cycle, often with other behaviors overlaid (e.g., searching-related hyperextensions, lateral 
turning, and dorsal-ventral undulations). Importantly, after a pause in the elongation phase, the movement is often completed from the start of the initial pause (Cacciatore et al., 2000). This flexibility resembles that observed during walking of the stick insect (Gruhn et al., 2009), cat (Akay et al., 2006), and human (Ivanenko et al., 2009). Recently, we established that every segmental ganglion of the leech ventral nerve cord contains a complete crawl oscillator (CPG), which can be independently activated (Puhl and Mesce, 2008). Because of this anatomically favorable situation, we examined the nature of the coupling of these oscillators and determined the role of higher-order descending information to choreograph their activation and coordination.

\section{Materials and Methods}

Animals and physiological solutions. Adult medicinal leeches (Hirudo verbana) (Siddall et al., 2007) weighing between $1.5 \mathrm{~g}$ and $3.0 \mathrm{~g}$ were obtained from either Leeches USA or Niagara Medical Leeches. Leeches were maintained, at room temperature, in distilled water containing dilute Instant Ocean sea salts ( $1.89 \mathrm{~g} /$ gallon; Spectrum Brands).

Leeches were anesthetized in ice-chilled saline before dissection. All dissections and electrophysiological recordings were performed at room temperature in chilled normal saline. Normal saline contained (in $\mathrm{mm}$ ) 116.0 NaCl, $4.0 \mathrm{KCl}, 1.8 \mathrm{CaCl}_{2}, 1.5 \mathrm{MgCl}_{2}, 10.0$ dextrose, and 10.0 Trizma preset crystals, pH 7.4 (Sigma-Aldrich) (adapted from Nicholls and Baylor, 1968). To block chemical synaptic transmission, a $20: 1 \mathrm{Mg}^{2+}$ / $\mathrm{Ca}^{2+}$ ratio saline was used (mM): $87.0 \mathrm{NaCl}, 4.0 \mathrm{KCl}, 1 \mathrm{CaCl}_{2}, 20 \mathrm{MgCl}_{2}, 10.0$ dextrose, and 10.0 Trizma preset crystals, pH 7.4 (Szczupak et al., 1998). Saline containing dopamine (DA) was made fresh daily and used within $5 \mathrm{~h}$. To obtain final DA concentrations of 50-100 $\mu \mathrm{M}$, a $1 \mathrm{mM}$ DA stock was diluted with normal saline immediately before use (Puhl and Mesce, 2008).

Overview of leech neuroanatomy and nomenclature. The nervous system of the leech consists of a compound cephalic ganglion (i.e., the supraesophageal and subesophageal ganglia), 21 segmental ganglia (designated M1-M21), and a compound tail ganglion (see Fig. 4A). Connectives joining these ganglia are comprised of two hemiconnectives and a smaller median Faivre's nerve (FN).

Each segmental ganglion contains a dorsal and ventral layer of neuronal somata. Key somata of uniquely identifiable motoneurons (MNs) active during crawling are the ventral lateral circular excitor (CV) MNs and the dorsal longitudinal excitor (DE-3) MNs, located on the ventral and dorsal surfaces, respectively (Stuart, 1970).

Experimental preparations and nomenclature. In this study, locomotor behaviors were monitored in both nearly intact and isolated CNS preparations. The nearly intact preparation allowed for observation of overt locomotion while providing direct access to the CNS (Esch et al., 2002). The term "isolated whole nerve cord" refers to a complete CNS preparation dissected from the body, while the term "chain preparation" refers to a subset of isolated ganglia lacking the cephalic and tail ganglia. To observe fictive locomotion in isolated or chain CNS preparations, motor patterns were monitored by recording the extracellular activity of DE-3, whose large axon resides in the dorsal posterior (DP) nerve roots of each ganglion. The DE-3 spike is typically the largest unit in the DP recordings (Kristan et al., 1974). We defined fictive crawling as three or more consecutive bursts of DE-3 with periods ranging from 5 to $25 \mathrm{~s}$ (Eisenhart et al., 2000; Puhl and Mesce, 2008).

For isolated whole nerve cord preparations, the entire intact CNS was removed with both left and right DP nerves from 2-4 segmental ganglia. Shorter chain preparations were made by removing 4-6 segmental ganglia, ensuring the connectives remained intact. These chains were taken from M7-M18, including both left and right DP nerves from at least 2-3 segmental ganglia. When necessary, a Vaseline well was placed around individual ganglia, isolating them for focal DA application. In some experiments, the FN was manually severed or crushed using a fine forceps, leaving both hemiconnectives intact. The hemiconnectives were severed by cutting them with fine-tipped scissors, leaving only the FN intact.

Briefly, for the nearly intact preparation, the body tissues surrounding the cephalic ganglion through ganglion M3 were removed while keeping the remaining body intact. The nerve roots of the tail ganglion and M4 were severed. The preparation was stabilized in a two-chambered dish by anchoring the anterior edge of the body wall to a wax pad with the intact portion of the animal placed in the larger posterior chamber, leaving it free to locomote (see Fig. 2 A, schematic). The fluid levels of the posterior chamber could be changed from shallow $(<0.1 \mathrm{~cm})$ to deep $(1.0 \mathrm{~cm})$. We observed spontaneous crawling, as it was produced by the nearly intact leech, or we elicited crawling by gently pulling on the rear of the leech with a small applicator stick to elongate its body, which was shown previously to produce crawling reliably (Esch et al., 2002). The applicator stick was gently applied to the dorsal aspect of the body to elicit swimming.

Sucrose block, ion exchange block, and focal $\mathrm{K}^{+}$-induced excitation. We used the sucrose block technique of Masino and Calabrese (2002) to reversibly block signals descending from the cephalic ganglion in nearly intact and isolated whole nerve cords. The sucrose delivery system was modified by constructing a bow-tie-shaped well made of Sylgard (Dow Corning), which was placed across the connectives between two ganglia (M2-M3). Neural transmission was blocked when the well's saline was replaced with an isotonic sucrose solution $(260 \mathrm{~mm})$ that lacked the ions necessary for neural transmission (e.g., $\mathrm{Na}^{+}, \mathrm{K}^{+}, \mathrm{Ca}^{2+}$ ). In our hands, we confirmed the effectiveness of the sucrose block by demonstrating that neural transmission was eliminated in sucrose and restored when the well was replenished with normal saline (Fig. 1).

To block chemical synaptic activity within a single ganglion while not disrupting fibers of passage, a given ganglion was isolated within a Vaseline well and bathed for 15-20 min in a saline solution containing high $\mathrm{Mg}^{2+}$ and low $\mathrm{Ca}^{2+}$ (recipe provided above). Similar methods have been used in the leech and crayfish to silence individual segmental CPGs for locomotion (Pearce and Friesen, 1985; Tschuluun et al., 2001). To increase the general excitation of the cephalic ganglion, a $2 \mathrm{M} \mathrm{K}^{+}$acetate $/ 20 \mathrm{~mm} \mathrm{~K}^{+}$chloride solution was briefly applied; a similar method was used by Friesen and Brodfuehrer (1984) to depolarize cephalic leech neurons. The solution was washed away within several seconds of application by flow of the saline-containing superfusion system.

Electrophysiological recordings and cell identification. Extracellular unit activity was recorded from a chosen DP nerve using suction electrodes 

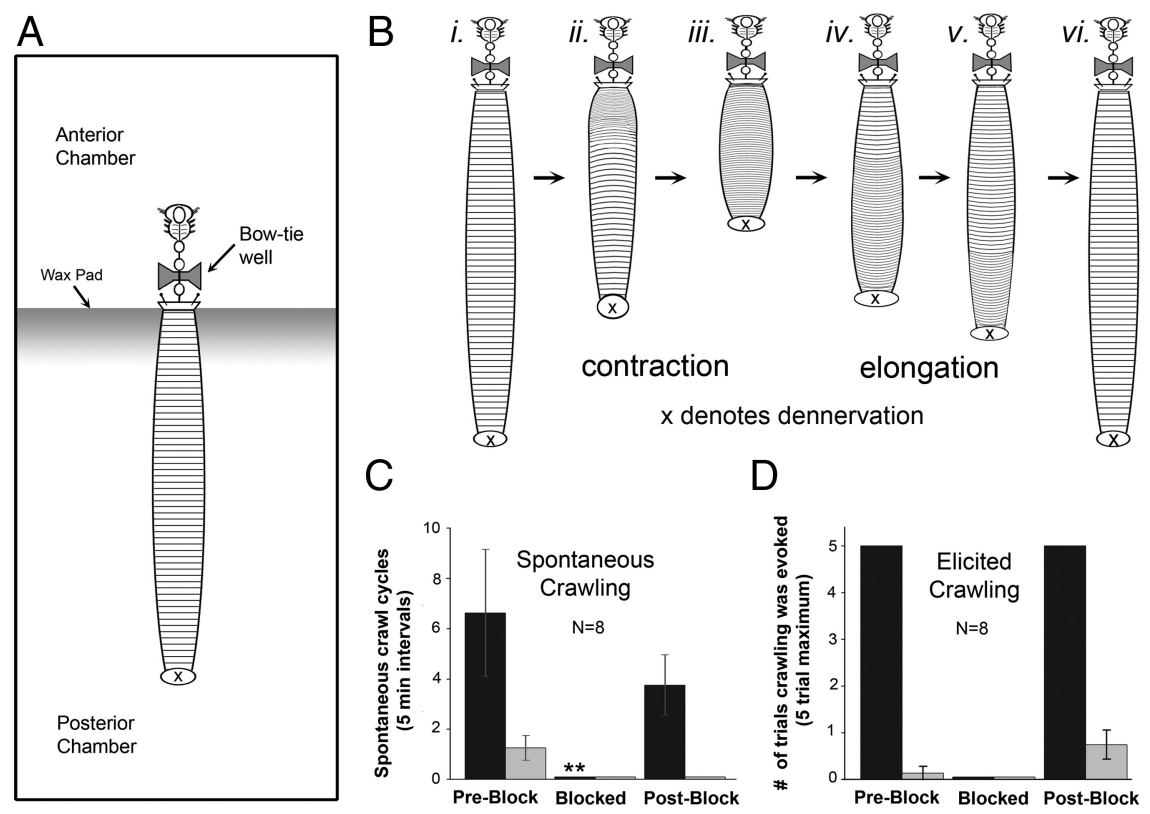

( $\sim 50 \mu \mathrm{m}$ tip width). Extracellular signals were amplified using a Grass Technologies P15 AC preamplifier and recordings were digitized (10,000 samples/s) using a MacLab/4s data acquisition system (ADInstruments) attached to a Microsoft Windows-compatible personal computer. Activity of DE-3 in all DP recordings served as a monitor of fictive crawling. The intracellular activities of identified MNs were recorded using a Dagan IX2-700 amplifier and digitized as described above. Electrodes were filled with a solution of $2 \mathrm{~m}$ potassium acetate and $20 \mathrm{~mm}$ potassium chloride and had resistances of 40-65 $\mathrm{M} \Omega$. For cell labeling, intracellular pipette tips were back-filled with a 2 $\mathrm{M}$ potassium acetate solution that contained $5 \%(\mathrm{w} / \mathrm{v})$ Neurobiotin (Vector Laboratories).

MNs were identified by their electrophysiological signatures and the position and size of their somata. Cell identities were confirmed by iontophoretically injecting 5\% Neurobiotin (0.5-1 nA) into a given cell for 10-30 min. Tissues were fixed, processed, treated with 1:50 Cy3-conjugated Streptavidin (Jackson ImmunoResearch) in PBS for $24 \mathrm{~h}$ at $4^{\circ} \mathrm{C}$, and prepared for viewing according to the methods of Gilchrist et al. (1995).

Figure 2. Crawling behavior in the nearly intact leech and its dependence on descending signals from the cephalic ganglion. $\boldsymbol{A}$, Schematic drawing of the nearly intact leech preparation. A bow-tie-shaped well was placed across the connectives between $M 2$ and $M 3$. The well was sequentially filled with saline, isotonic sucrose, and then saline to restore neural transmission. $\boldsymbol{B}$, Schematic drawing of overt crawling behavior in nearly intact leech preparations when descending signals were not blocked. $\boldsymbol{i}$-iii indicate the contraction phase and $\boldsymbol{i v}-\boldsymbol{v i}$ the elongation phase of one overt crawl cycle. C, Number of spontaneous crawl cycles observed for 8 animals during a 5 min interval for each condition (i.e., preblock, blocked, postblock). D, Number of trials (out of 5 ) that crawling was elicited in 8 animals after a gentle hyper-elongation of the body; same three conditions were tested. $C, D$, Black bars represent shallow water ( $<1 \mathrm{~mm}$ depth), and gray bars represent deep water $\left(10 \mathrm{~mm}\right.$ depth). ${ }^{* *} p<0.01$. Error bars are \pm SEM.

Data analysis and statistical methods. To determine the temporal properties of fictive crawling, we analyzed bursting activity of DE-3. A grouping of DE-3 spikes was deemed a burst if a minimum of 10 spikes were separated by $<2$ s, although most bursts contained dozens of spikes. Crawl periods were calculated by measuring onsets of sequential DE-3 bursts. If

\section{Swimming in Nearly-Intact Preparations $(\mathrm{N}=8)$}

A

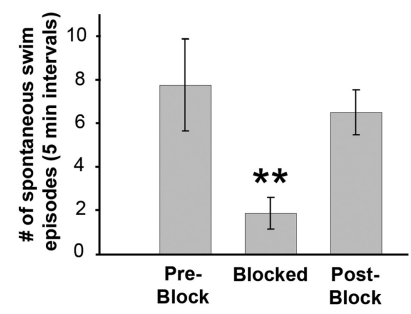

C

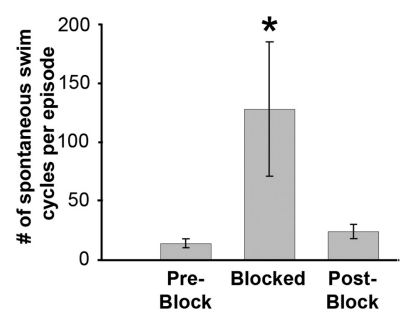

B

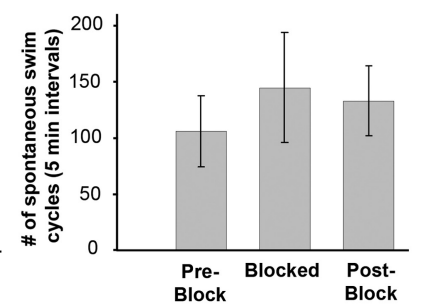

$\mathrm{D}$

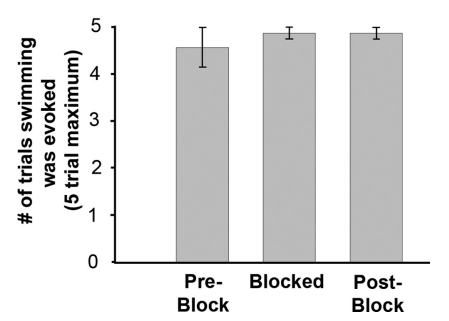

Figure 3. Swimming behavior in the nearly intact leech and its lack of dependence on descending signals. $\boldsymbol{A}$, Number of spontaneous swim episodes (uninterrupted bouts of swimming) in a 5 min interval for each condition (preblock, blocked, postblock). Overt swim bouts were easily discerned as the animal did not pause between successive swim cycles. $\boldsymbol{B}$, Total number of spontaneous swim cycles in a 5 min interval for each experimental condition. $C$, Number of spontaneous cycles per swim episode for each experimental condition. $\boldsymbol{D}$, Number of trials (out of 5 ) in which swimming was elicited after gently stroking the dorsal body surface. All bars represent swimming in high water conditions (10 $\mathrm{mm}$ depth). No leeches swam in low water $\left(<1 \mathrm{~mm}\right.$ depth). ${ }^{*} p<0.05,{ }^{* *} p<0.01$. Error bars are \pm SEM. $n=8$ leeches. burst start times were not clearly discernable ( $<15 \%$ of samples), burst midpoints were used to calculate the period. To determine midpoints, DE-3 spike frequency over time was calculated by counting the number of DE-3 spikes per $250 \mathrm{~ms}$ time bin and multiplying by 4 bins/s (spikes/s). For each preparation, a spike frequency threshold was calculated by adding one SD of the DE-3 spike frequency to the minimum spike frequency observed during the analysis window. Burst start and end times were when the spike frequency crossed threshold. The midpoint of each burst was calculated by adding 0.5 times the burst duration to the burst start times. Mean crawl periods were determined by analyzing 6-10 consecutive crawl cycles. Intersegmental delays (ISDs) were determined by subtracting posterior DE-3 onsets from anterior ones and dividing by the number of segments separating the two. Mean ISDs were determined by analyzing 5-13 consecutive crawl cycles. Burst midpoints were used to calculate ISDs in lieu of burst start times if burst onsets were not clearly discernable. Intersegmental phase delays, in degrees per segment, were calculated by multiplying the ISD by $360^{\circ}$ and dividing by the corresponding crawl period. For ISD and phase delay calculations, the most anterior DP recordings were used as the reference signal.

The Mann-Whitney-Wilcoxon (MWW) nonparametric test was used to assess whether two independent samples came from the same distribution and was performed using the free software package R (R Development Core Team, 2009). All statistical tests were two-tailed with a $95 \%$ confidence level $(\alpha=0.05)$. Sample means were presented \pm the SEM.

\section{Results}

Descending signals from the cephalic ganglion are necessary for overt crawling in nearly intact leeches

Figure 1 demonstrates the effectiveness and reversibility of the sucrose block. By placing this block below the cephalic ganglion, we determined the necessity of cephalic signals for overt crawling behavior in nearly intact leech preparations (Fig. 2A,B). Figure $2 B$ depicts the contraction and elongation phase of one overt 
crawl cycle. Spontaneous crawling behaviors were monitored when the body of the leech was situated in either shallow or deep water, and measured before the sucrose blockade (preblock), during the block (blocked), and after the sucrose was exchanged with normal saline (postblock). We observed no spontaneous overt crawling during the block in either shallow (black bars) or deep (gray bars) water (Fig. 2C). In shallow water, the number of spontaneous crawl cycles was significantly less than in the preblock and postblock conditions $(p<0.01)$ (Fig. $2 C$ ). Spontaneous crawling in deep water was limited and only observed during the preblock phase (Fig. 2C). Although these data indicate that descending cephalic signals are necessary for spontaneous overt crawling, they did not establish whether the absence of these signals rendered leeches incapable of executing crawling when stimulated. To study this possibility, we attempted to elicit crawling by gently hyperelongating the posterior body with a small applicator stick (Esch et al., 2002). During the preblock phase, in shallow water, crawling was elicited in all animals $(n=8)$ in $100 \%$ of trials ( 5 of 5 attempts) (Fig. 2D). During the blocked phase, it was not possible to elicit a single cycle of crawling in any of the animals tested (Fig. 2D). During this phase, we attempted to elicit crawling by pricking the cuticle with a metal pin just anterior to the tail sucker $(n=3)$. Under this noxious stimulation, $100 \%$ of preparations failed to crawl, although the stereotypic shortening response was exhibited (Kristan et al., 1982) (data not shown). During the subsequent postblock phase, in shallow water, crawling was stimulated in 5 of 5 attempts among all animals tested (Fig. 2D). These data provide clear evidence that descending signals from the cephalic ganglion are necessary for the expression of overt crawling in nearly intact animals.

\section{Crawling and swimming are differentially dependent on descending signals from the cephalic ganglion}

In this companion study, we determined whether the removal of descending signals also prevented swimming. Although a number of previous reports had shown that swimming can occur in the absence of the cephalic ganglion, they involved surgical manipulations that were irreversible (Brodfuehrer and Friesen, 1986a; Cornford et al., 2006). Thus, we analyzed spontaneous and evoked overt swimming behavior using the reversible sucrose block. We conducted our analysis in shallow and deep water using the same animals analyzed in the previous section. Because no leeches swam in shallow water, data only for the deep water condition were plotted (Fig. 3).

Spontaneous swimming was observed in all animals tested in all three conditions (Fig. $3 A-C)(n=8)$. The number of swim
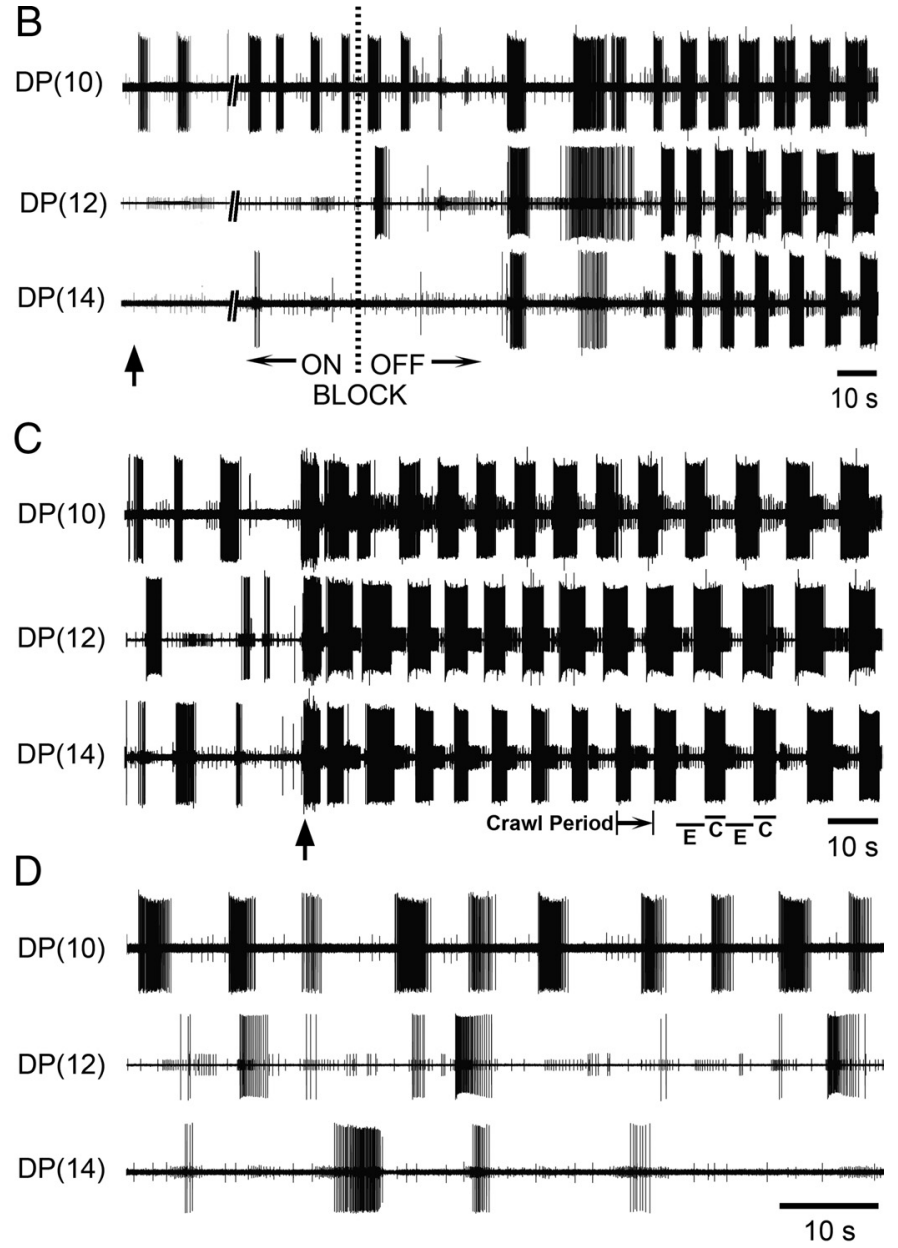

$10 \mathrm{~s}$

Figure 4. Cephalic descending signals are necessary and sufficient for appropriate intersegmental coordination during DAbiased fictive crawling. $A$, Schematic drawing of the whole nerve cord and placement of the bow-tie-shaped well containing saline block OFF) or isotonic sucrose (block ON). Locations of extracellular recordings are marked by electrodes on 3 evenly spaced dorsal (DP) nerves (M10, M12, and M14). Site of $\mathrm{K}^{+}$excitation is indicated at the left of the cephalic ganglion. $\boldsymbol{B}$, Initial record , Expanded trace of DP nerve activity when the sucrose block was in effect. Previous crawling exhibited before this block $(\boldsymbol{C})$ was replaced with uncoordinated DE-3 bursting.

episodes (bouts) observed during the blockade of cephalic signals was significantly different from the preblock and postblock phases $(p<0.01)$ (Fig. $3 A$ ), although no significant differences in the total number of swim cycles was found between the three phases $(p>0.5)$ (Fig. $3 B$ ). It is noteworthy that the number of swim cycles within an individual swim episode was significantly larger $(p<0.05)$ during the blocked phase (127.9 \pm 57 cycles per episode) than during the preblock and postblock phases (Fig. 3C) (13.9 \pm 3.8 and $24 \pm 5.9$ cycles per episode, respectively).

To examine evoked swimming, we gently stroked the dorsal surface of the leech's body. In nearly all attempts (out of 5), swimming was elicited during the preblock, blocked, and postblock phases (Fig. 3D). These data demonstrate that crawling and swimming are differentially dependent on descending cephalic signals, and are consistent with a previous report indicating that the cephalic ganglion may play a predominantly suppressive role 
A

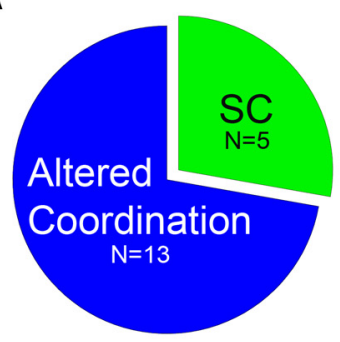

B Pre-Block

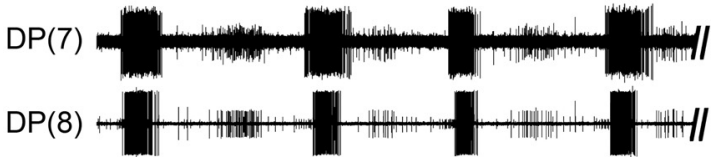

Blocked

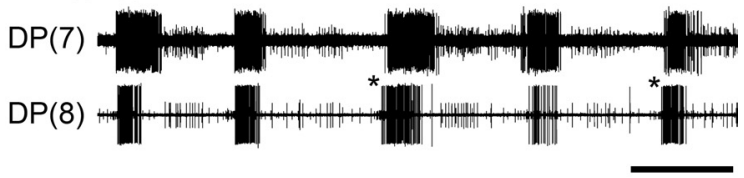

$10 \mathrm{~s}$

C Pre-Block

Blocked

$\mathrm{DP}(9)$

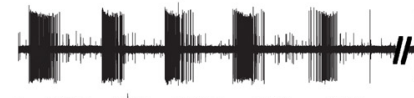

DP(11)
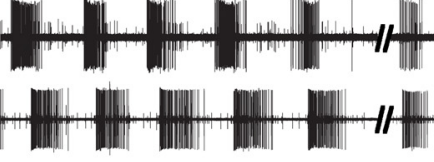

$\mathrm{DP}(13)$

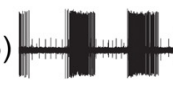

$\mathrm{D}$

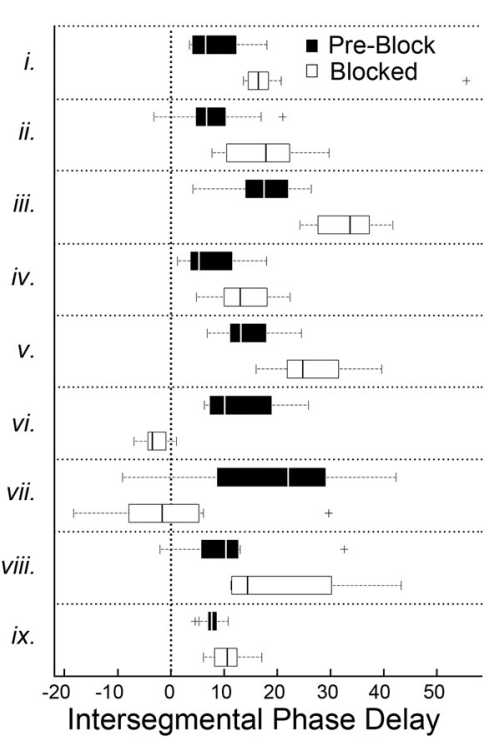

(degrees/segment)
E
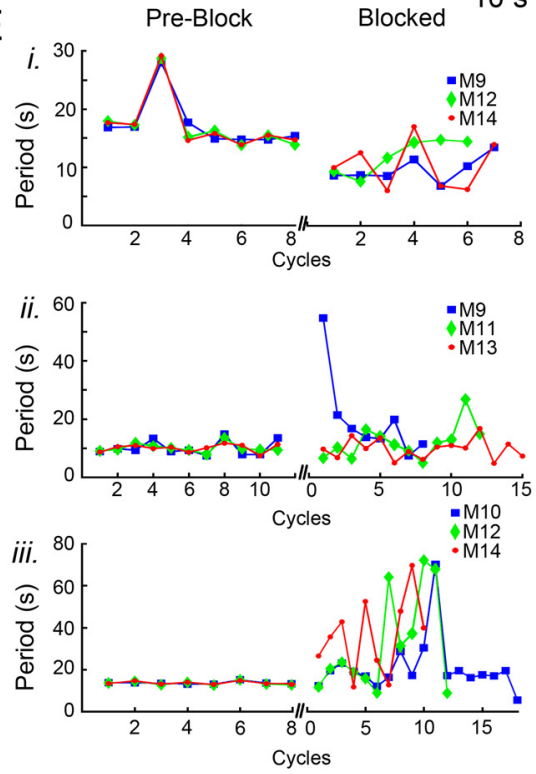

Figure 5. In whole isolated nerve cords, sucrose blockade of cephalic descending signals abolishes the posterior-directed intersegmental coordination of the DA-biased crawl oscillators. $A$, The number of preparations that stopped crawling (SC) and those that had altered coordination during the sucrose block. $B$, DP recordings of fictive crawling in two adjacent segments before the block (preblock) and during it (blocked). Intersegmental delays (ISDs) were altered during cephalic blockade and intersegmental phase advances (asterisks) became evident (note: rare in controls). C, DP nerve activity from 3 segments showed robust and coordinated fictive crawling in preblock (left). Shortly after blocking descending inputs (blocked, right traces), uncoordinated DE-3 bursting emerged; this preparation exemplifies those with variable periods. $\boldsymbol{D}$, Box plots (black $=$ preblock; white $=$ blocked) summarizing changes in DE-3 intersegmental phase delays for preparations showing no significant change in cycle period $n=9$, labeled $\boldsymbol{i}$-ix). Plus symbols denote outlier data points. $\boldsymbol{E}$, Fictive crawl periods obtained from 3 segmental oscillators plotted over successive cycles in preblock and again when cephalic inputs were removed (blocked) $(n=3$ whole nerve cord preparations labeled $\boldsymbol{i}$-iii). Before the block (plots at left), the outputs from all 3 oscillators exhibited the same period. During the blockade (plots at right) periods were no longer stable and varied across all 3 segments. Data for cycle periods during the block were obtained within the following time windows: $74 \mathrm{~s}(\boldsymbol{i})$, $158 \mathrm{~s}$ (iii), and $381 \mathrm{~s}$ (iii); thus some cycles have only 1 or 2 symbols shown.

in controlling swimming behavior (Brodfuehrer and Friesen, 1986a).

Cephalic descending signals are necessary and sufficient for coordinated fictive crawling biased by dopamine

Based on the results of the previous section, we hypothesized that descending inputs provide multiple levels of regulation: (1) a system-wide activation and maintenance of the independent segmental crawl oscillators and (2) the setting of the intersegmental

phase relationships during each crawl cycle. To understand the specific contributions of descending inputs for oscillator coordination, we needed first to bias the CNS in the direction of crawling while simultaneously suppressing swimming so that these questions could be addressed. This strategy was best achieved by applying 50-100 $\mu \mathrm{M}$ DA (Crisp and Mesce, 2004; Puhl and Mesce, 2008) to isolated whole nerve cords and monitoring fictive crawling via 2 or more DP nerves separated by a given number of segments (Fig. $4 A$ ). We again used the sucrose block to reversibly remove cephalic descending signals; in addition, in some preparations we focally stimulated the cephalic ganglion to initiate fictive crawling.

In Figure $4 B$, we show activity of the crawl-monitor cell, DE-3, across multiple ganglia (DP nerves 10,12, and 14) in the absence (block $\mathrm{ON}$ ) and then presence (block OFF) of cephalic descending inputs. The isolated whole nerve cord was biased to crawl by applying $50 \mu \mathrm{M}$ DA, a concentration at the threshold for inducing crawling (Puhl and Mesce, 2008). At the beginning of the recordings shown (Fig. $4 B$, far left arrow), the cephalic ganglion was depolarized with $\mathrm{K}^{+}$while the sucrose block was in effect; note the lack of any changes in recorded activity. When the block was subsequently removed (Fig. 4 B, block OFF), robust and coordinated fictive crawling emerged across all three segments. In a complementary experiment, we stimulated the cephalic ganglion focally with $\mathrm{K}^{+}$(Fig. 4C, arrow) to demonstrate that cephalic activation is sufficient to induce coordinated fictive crawling when descending inputs are present (note: DE-3 bursts showed no intersegmental coordination before cephalic stimulation). This stable and robust metachronal crawling was subsequently abolished in the presence of the block (Fig. 4D).

\section{Analysis of crawl disruption when cephalic inputs were removed}

In isolated whole nerve cord preparations receiving the sucrose block, DA-induced fictive crawl-like bursts were either intermittent or ceased altogether [i.e., stopped crawling (SC) $(n=5)]$ or exhibited altered intersegmental coordination $(n=$ 13) (Fig. 5A). The removal of cephalic inputs resulted in a spectrum of changes whereby ISDs changed but cycle periods remained fairly constant (Fig. $5 B)(n=9 / 13)$ or periods changed within and between segments (Fig. $5 C)(n=4 / 13)$, all of which abolished the metachronal intersegmental phase relationships. During experiments in which recorded crawl bursts could readily be discerned across all recorded segments (e.g., Fig. 5B), intersegmental phase delays were calculated and differed significantly in preblocked and blocked conditions (Fig. 5D). Statistical signifi- 
cance between intersegmental phase delays of preblock and blocked conditions was determined for each preparation. In preparations that showed more pronounced alterations (Fig. $5 C)$, changes in intersegmental coordination were assessed by measuring the period of DE-3 crawl bursts within and across segments. Figure $5 E$ shows changes in the intrasegmental and intersegmental periods for 3 different preparations ( $i-i i i)$ before and during the sucrose block. During preblock conditions, all 3 nerve cords revealed isochronicity (i.e., a fairly constant period), and all crawl oscillators within a preparation exhibited a similar crawl period (Fig. 5E, note flat and overlapping lines). Figure $5 E i$ illustrates that even when one crawl period transiently deviated from its set point (in preblock), all 3 segmental crawl oscillators shared the same deviation. Upon removal of descending signals, however, the stability of these periods was lost within and across the segmental oscillators (Fig. 5E, blocked). This block-induced variability in crawl period precludes the formation of appropriate phase delays and intersegmental coordination. In addition, we calculated the correlation of ISDs and period length for $80 \mathrm{crawl}$ cycles ( $n=8$ preparations) in control leeches and found a coefficient of correlation to be 0.73 . This result indicated that the ISD increases with cycle period. For overt crawling, Stern-Tomlinson et al. (1986) reported that ISDs also increase as crawl periods lengthen. Even if one were to argue that the loss of cephalic inputs translates into a decline in excitatory drive and slower crawl period, theoretically ISDs should still be able to adjust to changes in crawl period, but as shown in Figure $5 C$ the ISDs differ over successive cycles, indicating a disruption in coordination.

Finally, we tested whether removal of ascending information from the compound tail ganglion affects the intersegmental coordination of crawling. We used the sucrose block to suppress signals from the tail ganglion. We induced sustained fictive crawling by treating whole nerve cords with 75-100 $\mu \mathrm{M}$ DA and observed that blockade of ascending signals had no significant effects on the ISDs and periods of fictive crawling (data not shown; $n=4$ ).

\section{Crawl oscillators within chains of ganglia: lack of} intersegmental coordination and stability

In our previous study (Puhl and Mesce, 2008), we demonstrated that a single segmental ganglion can express robust and stable fictive crawling when treated with DA. Crawl periods within and across ganglia were similar (Puhl and Mesce, 2008). Based on this information, one might predict that a DA-treated chain of only a few ganglia would reveal more stable crawling; furthermore, as isolated chains they would provide yet another test of the need for cephalic descending signals to coordinate the iterated oscillators.

We observed that a DA-treated chain of 5 ganglia, lacking the cephalic ganglion, never exhibited coordinated crawling with appropriate intersegmental phase delays (Fig. 6A) $(n=5)$. Although each segmental crawl oscillator gave rise to DE-3 bursting in each segment (Fig. 6A), the intrasegmental and intersegmental timing events never mirrored those in the isolated ganglion or whole nerve cord. Thus, the crawl oscillators appear to be most stable either in complete isolation or in the presence of intact descending inputs. One hypothesis is that any intermediate variants in input perturb the crawl system as a whole. An alternative hypothesis is that the crawl oscillators in chains lack sufficient descending cephalic drive needed to activate DA-biased crawling. To test these ideas further, we removed the end ganglion (in the chain shown in Fig. 6A) so that it was freed from all intersegmental inputs (Fig. 6B, left diagram). As predicted by our first hy-
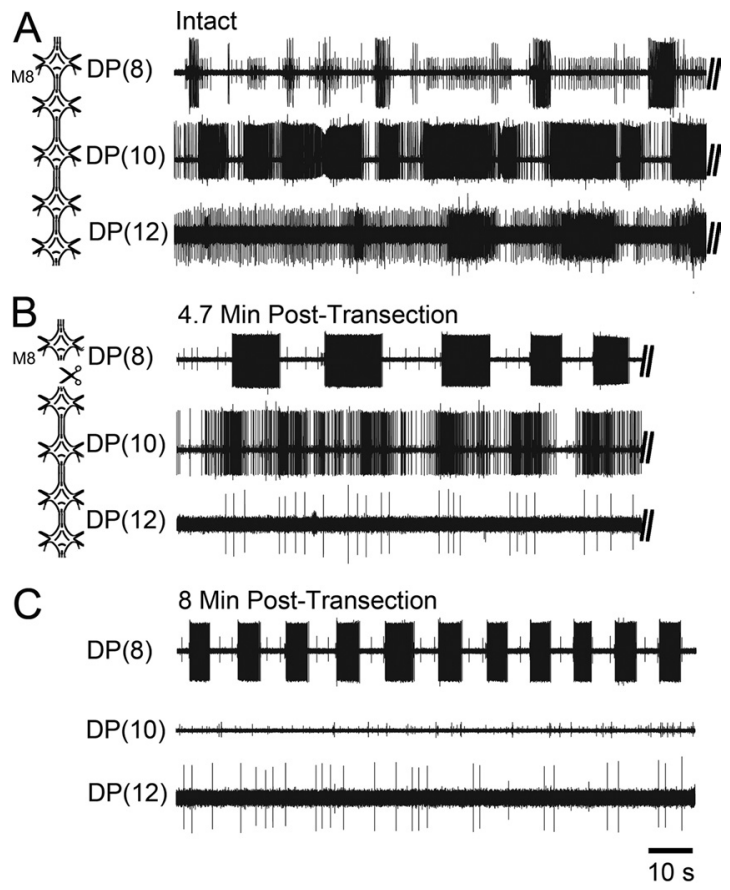

Figure 6. In the absence of cephalic inputs, a chain of DA-biased ganglia reveals uncoordinated and unstable $\mathrm{DE}-3$ bursting, but a single ganglion isolated from this chain transitions to stable and robust fictive crawling. $\boldsymbol{A}$, Schematic of a chain of 5 ganglia (M8-M12) (left) and motor activity recorded from DP nerves 8,10 , and 12 (right). Recordings revealed the lack of robust rhythmic DE-3 bursting and showed an inappropriate metachronal wave of intersegmental DE-3 activity. $\boldsymbol{B}$, Left, Schematic showing the physical isolation of one ganglion (M8) from the chain. Right, DP nerve activity from the same preparation as in $A 4.7 \mathrm{~min}$ after transecting the M8 -M9 connectives. DE-3 bursting in the isolated ganglion became more robust and burst structure solidified; unstable bursting activity in DP(10) continued. C, DP recordings $8 \mathrm{~min}$ after transection showing the emergence of robust fictive crawling [DP(8)] with stable shorter crawl periods (top trace). DE-3 bursting in the other two ganglia was absent (middle and bottom traces).

pothesis, we found that a strong crawl rhythm emerged (Fig. $6 \mathrm{~B})$; it became even more robust and stable over time in isolation (Fig. 6C).

\section{Role of individual oscillators for intersegmental crawl drive} At this juncture, it is clear that the cephalic ganglion plays a vital role in coordinating the segmental crawl oscillators, thus generating the posterior-directed metachronal waves of motor activity during each crawl cycle. Furthermore, DA-induced crawling is most unstable when only a subset of intersegmental signals is present, but Figure $6 \mathrm{~A}$ also hints at the idea that the crawl oscillators influence the cycling frequency of nearby crawl oscillators. To begin to identify shorter-distance intersegmental signals, we asked whether individual crawl oscillators are capable of influencing or driving crawl-like activity in neighboring ganglia.

To address this question, we recorded DP nerve activity in chains of 4-6 ganglia from either the middle (M7-M14) or posterior (M15-M20) body regions, and exploited the ability of DA to activate a single crawl oscillator; we then measured the influence of one oscillator on its segmental neighbors. Figure $7 \mathrm{~A}$ shows an experiment in which a single ganglion (M13, shaded in gray) was induced by DA to express fictive crawling. The DP nerves of three ganglia were monitored simultaneously: the DAtreated ganglion (denoted G/DA) and the anterior $(\mathrm{G} /-1)$ and posterior $(\mathrm{G} /+1)$ adjacent ganglia (Fig. $7 A$, diagram at left). Before DA treatment, rhythmic DE-3 bursting was absent in all 

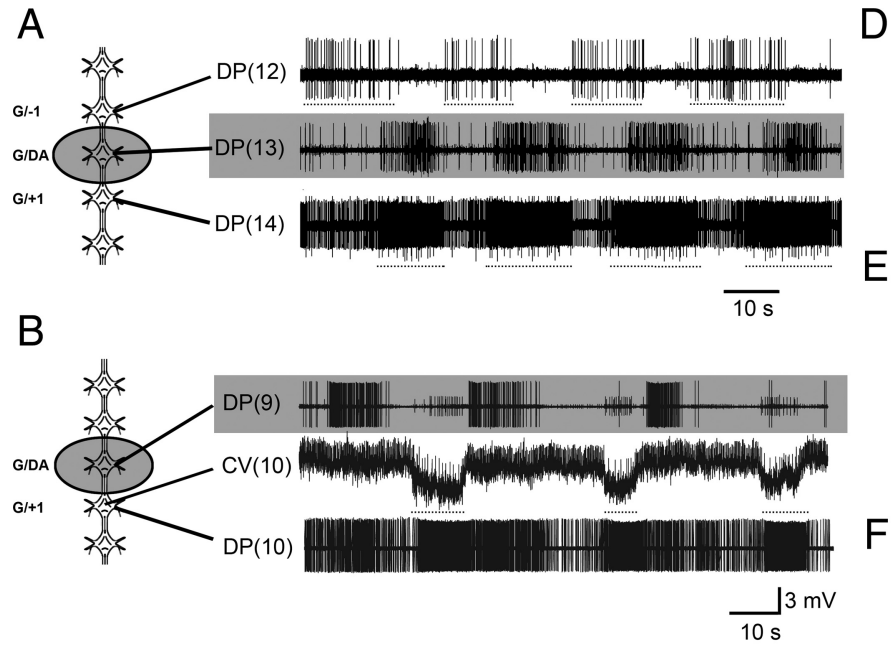

C
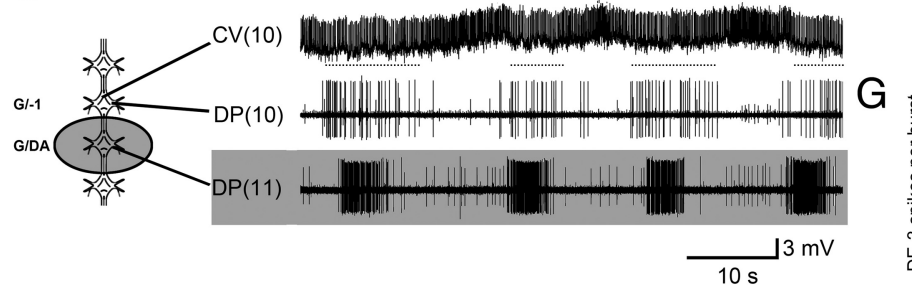

Figure 7. Ability of a single DA-activated crawl oscillator to drive crawl-like activity in adjacent ganglia. $\boldsymbol{A}-\boldsymbol{C}$, Left, Schematic drawings indicating site of DA placement (shaded well; $100 \mu \mathrm{M} \mathrm{DA}$ ) and specific ganglia recorded. Shaded records correspond only to those ganglia treated with DA. $A$, The DA-treated ganglion (G/DA, middle trace) expressed fictive crawling, which promoted crawl-like $D E-3$ bursting activity (dotted lines) in the adjacent anterior ganglion $(G /-1$, top trace) and posterior ganglion $(G /+1$, bottom trace). B, DA-induced fictive crawling [top trace, DP(9)] caused the untreated posterior ganglion [bottom trace, DP(10)] to show DE-3 burst-like activity. Furthermore, the intracellular record of the circular MN CV (middle trace) indicates that it is hyperpolarized (dotted lines) in phase with DE-3 excitation (bottom trace); an indicator of true fictive crawling. C, True fictive crawling, although notably quite weak, was also observed in the anterior ganglion (top and middle traces) in response to DA placed on its posterior neighbor (bottom trace); here CV was hyperpolarized (top trace, dotted lines) in phase with DE-3's depolarization (middle trace). $\boldsymbol{D}$, Percentage of preparations exhibiting DE-3 bursting in untreated ganglia located adjacent to ganglia induced to crawl by DA. $\boldsymbol{E}$, Percentage of preparations exhibiting anti-phasic activity in $\mathrm{CV}$ and DE-3 motoneurons. $\boldsymbol{F}$, Fictive crawl-like periods (i.e., DE-3 bursts) were similar across untreated ganglia located anterior $(G /-1)$ and posterior $(G /+1)$ to a given DA-treated ganglion $(G / D A)$. $G$, Number of DE-3 spikes/burst across untreated and DA-treated ganglia $(G /-1, G / D A, G /+1)$. Note that the number of $D E-3$ spikes/burst between $G /-1$ and $G /+1$ is very different. ${ }^{*} p<0.05$. Error bars depict $\pm S E M$. Numbers inside the bars of $\boldsymbol{D}-\mathbf{G}$ denote the sample sizes.

three DP recordings (data not shown). Shortly after DA was applied normal fictive crawling developed in the treated ganglion with a period of $\sim 22 \mathrm{~s}$ (Fig. $7 A$, shaded middle trace). In $\mathrm{G} /-1$ (not treated with DA), we observed distinct DE-3 bursts with regular cycle periods of $\sim 23 \mathrm{~s}$, resembling fictive crawling (Fig. $7 A$, top trace). The intraburst firing rate of DE-3, however, was lower than what would typically be expected in ganglia induced to crawl with DA (Puhl and Mesce, 2008). In G/ + 1, DE-3 was much more active, exhibiting a rhythmic modulation of its spiking frequency with a period of $\sim 22 \mathrm{~s}$ (Fig. $7 A$, bottom trace), but individual pseudo-bursts were not distinct. It is noteworthy that we did not observe normal intersegmental phase delays of DE-3 bursting in any of the DA-treated chain preparations we examined $(n=60)$. In some preparations, however, the burst frequencies of DA-treated and nontreated ganglia were similar.

Although the DE- 3 bursting in G/ -1 and G/ +1 resembled fictive crawling, several parameters needed to be fulfilled to substantiate this idea quantitatively. One key criterion to fulfill was that the circular (CV; elongation phase) and longitudinal (DE-3; contraction phase) MNs burst $\sim 180^{\circ}$ out of phase, as they do during DA-induced fictive (Puhl and Mesce, 2008) and overt

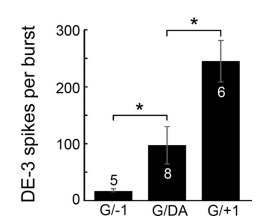

crawling (Gray et al., 1938; SternTomlinson et al., 1986). Figure $7 B$ shows a representative example of DE-3 (bottom trace) and CV (intracellular, middle trace) in $\mathrm{G} /+1$, showing that the spiking activity of CV decreased and the cell was hyperpolarized when DE-3 firing was elevated (note: this ganglion was not treated with DA). We also observed a similar antiphasic relationship of CV and DE-3 in G/ -1 (Fig. 7C). Note, however, that in $\mathrm{G} /-1$ the $\mathrm{CV}$ hyperpolarization is weak (Fig. 7C), suggesting that local crawl drive may be promoted less in the anterior direction and more in the posterior one.

Figure $7 D$ provides information about the total numbers and percentages of chain preparations in which DE-3 bursts were elicited in DA-treated and neighboring un-treated ganglia; as mentioned crawl-like bursts were more reliably observed in posterior directions. Figure $7 E$ shows the percentage of preparations showing anti-phasic $\mathrm{CV}$ and DE- 3 activity, and G/+1 again shows this feature more reliably than $\mathrm{G} /-1$. Furthermore, although the DE-3 crawl burst periods were indistinguishable across all segments and conditions (Fig. $7 F$ ), the number of DE-3 spikes/burst were significantly elevated in $\mathrm{G} /+1$ and fewer in G/-1 (Fig. 7G). All values were within normal range of fictive crawling (Eisenhart et al., 2000; Puhl and Mesce, 2008).

\section{The extent of local intersegmental drive}

To determine the potential extent of crawl-like drive to more distant ganglia, we examined DP nerve activity anteriorly in $\mathrm{G} /-2$, and posteriorly in $\mathrm{G} /+2$ and $G /+3$. Figure $8 A$ shows a record of DE-3 crawl-like bursting in G/+2 (bottom trace). Although many of the bursts across the 3 segments appear coactive, they lack appropriate phase delays. The percentage of preparations exhibiting crawl-like DE-3 bursting is shown in Figure $8 \mathrm{~B}$. No bursts resembling true fictive crawling were observed in $\mathrm{G} /-2$ (Fig. $8 B$ ).

To test whether the crawl drive from one segmental oscillator to another was based completely on a concatenated series of inputs from one segment to the next, we induced crawling in one ganglion (G/DA) and blocked chemical synaptic activity in the adjacent posterior ganglion with low- $\mathrm{Ca}^{2+}$, high- $\mathrm{Mg}^{2+}$ saline (blocked $\mathrm{G} /+1$ ) to determine whether crawl-like activity could still be communicated to $\mathrm{G} /+2(n=4)$. Figure $8 C$ shows the control chain of ganglia before treatment exhibiting spontaneous (tonic) activity in DE-3 across all 3 adjacent ganglia. In Figure $8 D$, fictive crawling was induced in $\mathrm{G} / \mathrm{DA}$ as shown (top trace). At this time, all neural activity was abolished in G/+ 1 (middle trace) in response to the low- $\mathrm{Ca}^{2+}$, high- $\mathrm{Mg}^{2+}$ solution (Fig. $8 D$ ). Although no neural activity was recorded from the intervening ganglion $(G /+1)$, the DA-induced crawling in G/DA was able to generate crawl-like bursting in $\mathrm{G} /+2$ (bottom 
A

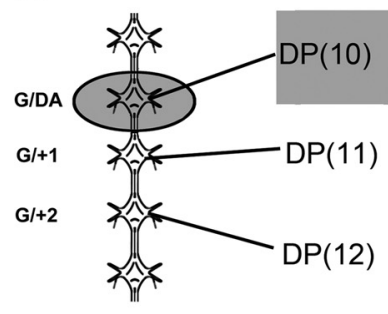

C

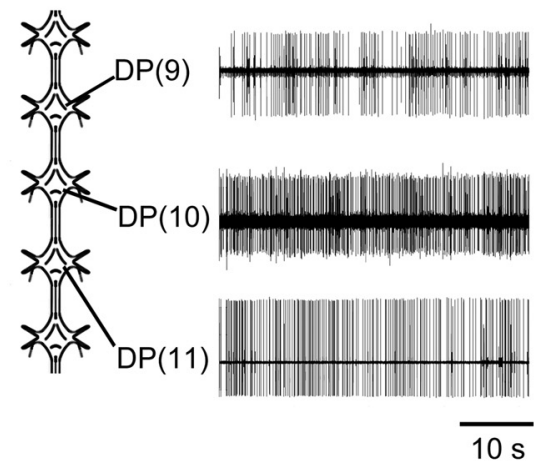

$\mathrm{B}$

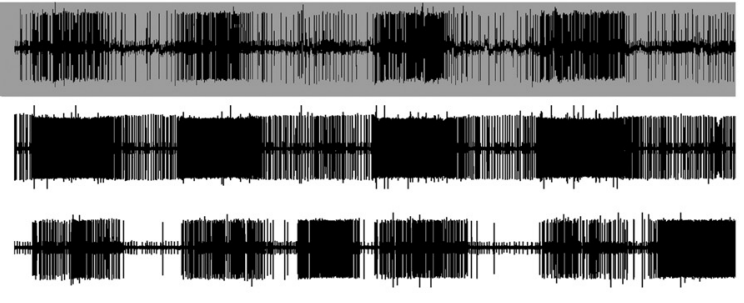

$10 \mathrm{~s}$

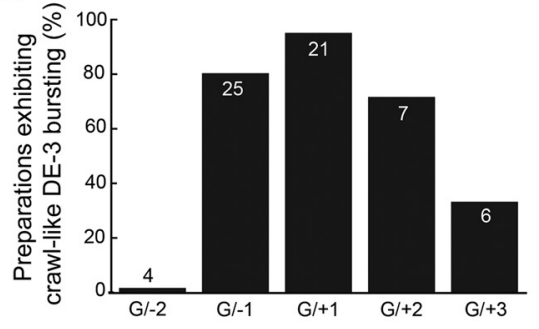

D

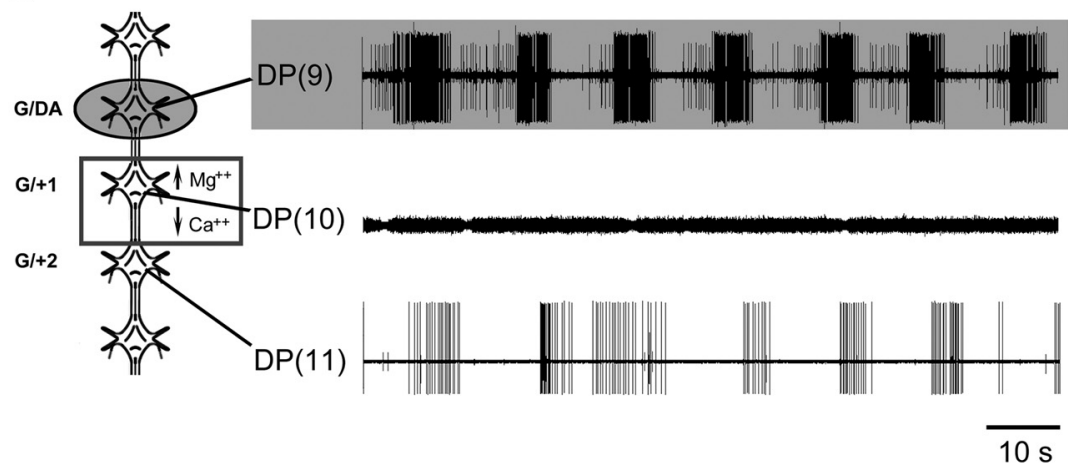

Figure 8. Crawl-like drive from an individual crawl oscillator extends to multiple posterior ganglia; signals can be carried via fibers of passage spanning two ganglia away from their source. $A, C, D$, Left, Schematic drawings indicating site of DA placement (shaded well; $100 \mu \mathrm{M} \mathrm{DA}$ ) and specific ganglia recorded. Shaded recording traces correspond only to those ganglia treated with DA. $A$, Fictive crawling in the DA-treated ganglion (top trace) and crawl-like activity driven in untreated ganglia up to two segments away (middle and bottom traces). $\boldsymbol{B}$, Percentage of preparations exhibiting $\mathrm{DE}-3$ bursting in response to $\mathrm{DA}$ on a given single ganglion $(\mathrm{G} / \mathrm{DA})$; drive extended up to 3 segments away $(\mathrm{G} /+3)$. Numbers inside bars denote sample sizes. $C$, Ganglia showing tonic $D E-3$ activity across 3 segments $[D P(9,10,11)]$ before treatment. $\boldsymbol{D}$, DP recordings from the same preparation as in $\boldsymbol{C}$ after DA-activated fictive crawling was induced (top trace) but chemical synaptic transmission was blocked in the adjacent posterior ganglion (middle trace). Box (left) surrounding ganglion $\mathrm{M} 10$ ( $\uparrow \mathrm{Mg}^{2+} / \downarrow \mathrm{Ca}^{2+}$ ) indicates procedure that resulted in no unit activity in DP(10) (middle trace). As shown in the DP(11) recording (bottom trace), DE-3 bursting was present, although weak, thus indicating that some crawl-like drive extended past this silenced ganglion.

trace), indicating that local intersegmental neurons must span a distance of 2 ganglia or more from their source.

\section{Local crawl-like drive is transmitted via} the hemiconnectives

As a first step toward identifying potential local crawl coupling and/or gating cells, we transected subcomponents of the intersegmental connectives to identify the anatomical route that carries the crawl-like drive. Three bundles of neuronal fibers comprise the connectives that link the individual segmental ganglia to each other: paired hemiconnectives and a singular (medial) FN. The hemiconnectives carry the vast majority of interganglionic fibers. The FN has been shown previously to carry an important gating cell for swimming, cell 204 (Weeks and Kristan, 1978), which conceivably could be multifunctional and help gate crawling between adjacent ganglia.

To determine which of these structures carries the fibers mediating the local crawl-like drive, we severed either the FN (Fig. $9 A$, far left diagram) or the hemiconnectives (Fig. $9 B$, far left diagram) between $\mathrm{G} / \mathrm{DA}$ and $\mathrm{G} /+1$. A 5-ganglia chain was used for both experiments and fictive crawling in a given ganglion was
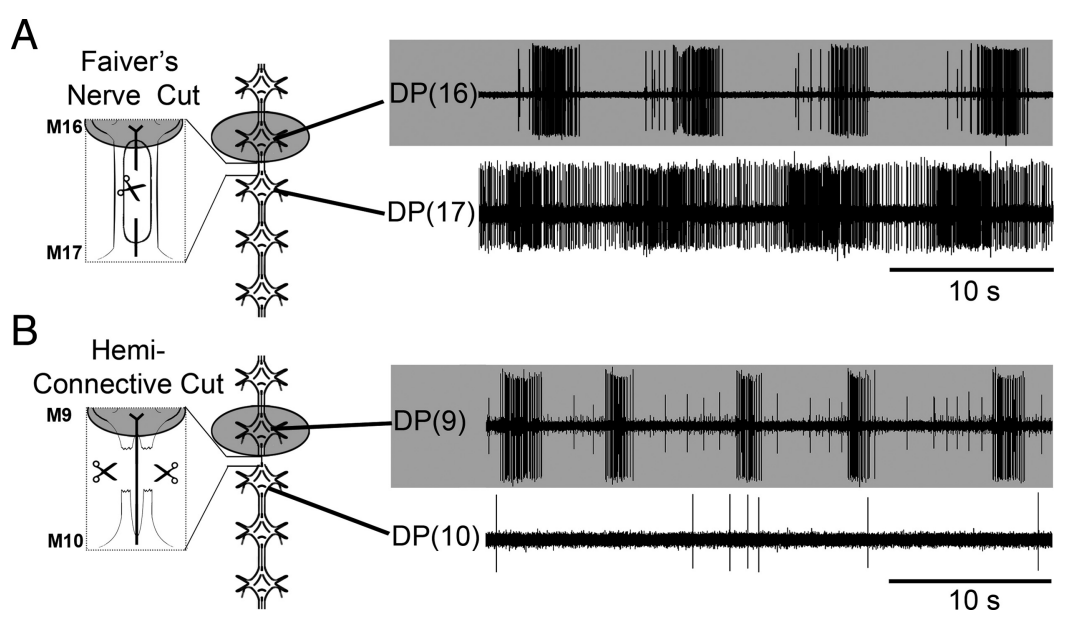

Figure 9. Testing the role of $\mathrm{FN}$ and the hemiconnectives in transmitting crawl-like drive to adjacent ganglia. $\boldsymbol{A}$, Left, Schematic drawing indicating site of DA placement (shaded well; $100 \mu \mathrm{M} \mathrm{DA}$ ), specific ganglia recorded, and location of cut Faivre's nerve. Right, Shaded recording corresponds to ganglion in DA (top trace) that exhibited robust fictive crawling [DP(16)]. Bottom trace shows crawl-like activity in adjacent posterior ganglion [DP(17)] after selective transection of the FN. B, Left, Schematic marking location of cut hemiconnectives. Right, Top trace shows DA-induced fictive crawling [DP(9)]. Bottom trace reveals the absence of any rhythmic $D E-3$ activity as a result of transecting the hemiconnectives.

activated with DA (G/DA in $100 \mu \mathrm{M})$. The recordings in Figure $9 A$ demonstrate that after severing the FN, crawl-like drive (bottom trace) was not disrupted (4 of 5 preparations). In contrast, when the hemiconnectives were severed in 5 different preparations, no crawl-like activity was recorded in $\mathrm{G} /+1$ (bottom trace) (e.g., Fig. 9B). 


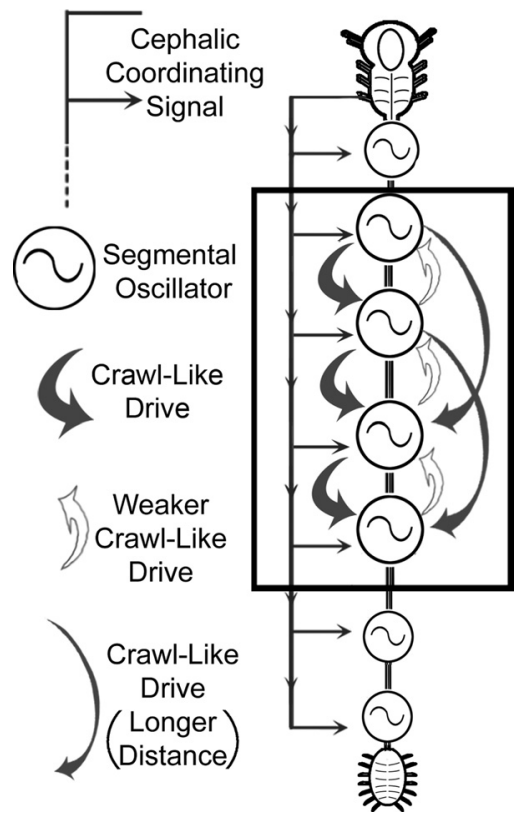

Figure 10. Schematic diagram of the leech CNS depicting neural elements involved in the intersegmental coordination of crawling. Each of the 21 midbody ganglia contains a complete crawl oscillator (circle with sigmoid) (Puhl and Mesce, 2008). The descending long-distance coordination signal(s) from the cephalic ganglion is necessary and sufficient for the production of normal intersegmental phase delays between the oscillators (continuous gray line with lateral arrows). In addition, individual crawl oscillators provide weak drive (open arrows) to their anterior neighbor and much stronger excitatory drive (thick gray arrows) to two or more oscillators in posterior locations. Intersegmental fibers of passage delivering crawl-like drive can project uninterrupted at least two ganglia away from their source (thinner gray arrows).

\section{Discussion}

\section{Higher-order descending information and local signals contribute to the intersegmental coordination of locomotor oscillators}

In this study of leech crawling, we demonstrated that the compound cephalic ganglion plays a critical role in both the activation and coordination of the segmental crawl oscillators; furthermore, we demonstrated that the individual locomotor oscillators provide excitatory drive to each other (Fig. 10, summary diagram). Although a number of computational models have been proposed to explain how long-distance projections might contribute to locomotion (Cohen and Kiemel, 1993; Cacciatore et al., 2000), to our knowledge, ours is the first empirical study to separate the contributions of higher-order and local drive, and intersegmental coordination.

The combination of descending and local control is likely tied to the flexibility inherent to leech crawling and, in some respects, parallels the adaptability observed for more complex locomotor behaviors like walking (Georgopoulos and Grillner, 1989; Lavoie et al., 1995). The dynamic nature of crawling is also based on oscillator independence (Puhl and Mesce, 2008). For productive locomotion, however, the coordination of these unit oscillators must be constrained so that an anterior-to-posterior wave of intersegmental activity is generated (Eisenhart et al., 2000; Puhl and Mesce, 2008). Because leeches do not crawl backwards nor exhibit intermittent positive and negative intersegmental phase shifts, the neural architecture of the animal must be organized to ensure that posterior-directed movements are executed. Our data indicate that information descending from the cephalic ganglion is necessary for the interoscillator phase delays that propagate in the anterior-to-posterior direction (Fig. 5). In addition, cephalic descending inputs are sufficient to organize appropriate intersegmental coordination when the individual crawl oscillators are biased to crawl by DA (Fig. 4). This higher-order coordination, likely in combination with local interoscillator coupling (Figs. 7, 8 ), acts to stabilize the intersegmental crawl periods and direct the rearward progression of intersegmental time delays during each phase (contraction or elongation) of the crawl cycle.

In other animal systems, studies have suggested that descending information is involved in oscillator coordination during locomotion. In walking cats (Shimamura and Kogure, 1983; Drew et al., 1986) and rodents (Gordon and Whelan, 2008), for example, descending signals from the midbrain and brainstem have been shown to contribute to interlimb coordination. Drew and Rossignol (1984) demonstrated that microstimulation of the medullary reticular formation in the thalamic cat, in addition to activating locomotion (Mori et al., 1978; Garcia-Rill and Skinner, 1987), can lead to phase-dependent changes in leg muscle activity that is incorporated into ongoing walking cycles.

\section{Oscillator-to-oscillator coupling}

Locomotor behaviors possessing greater stereotypy often appear less dependent on descending information for oscillator coupling. Although reticulospinal neurons in the lamprey, for example, can drive fictive swimming (McClellan and Grillner, 1984), coordinated swimming can be generated in isolated spinal networks if activated chemically or electrically (Cohen and Wallén, 1980). Such work underscores the importance of local oscillatorto-oscillator coupling.

Figures 7 and 8 demonstrate that individual crawl oscillators activated by DA can promote crawl-like activity in nearby oscillators. We further determined that crawl-like activity originating in a given ganglion extends to at least two posterior ganglia (Fig. $8 D$ ). These data suggest that this crawl-like drive is not limited to oscillator-to-oscillator interactions that are concatenated, but must also involve interneuronal signaling spanning multiple segments. These results parallel those in other systems in which well defined segmental oscillators communicate via intersegmental interneurons projecting through multiple segments (Pearce and Friesen, 1985, Tschuluun et al., 2001, Smarandache et al., 2009). Because this drive is stronger in the posterior direction, as evidenced by the increased number of spikes/DE-3 burst [proportional to muscle contraction (Mason and Kristan, 1982)] (Fig. $7 G$ ), the anterior-to-posterior propagation of crawl activity is facilitated. Importantly, this interoscillator signaling is not sufficient to produce normal intersegmental phase delays, thus local coupling provides only a supporting role for coordination, a feature that contrasts with the systems just discussed (Pearce and Friesen, 1985, Tschuluun et al., 2001, Smarandache et al., 2009).

Asymmetric oscillator coupling is a critical feature governing the directional propagation of motor activity in many locomotor systems; however, most studies have investigated this coupling in tightly coupled systems, often lacking the flexibility inherent in leech crawling. Swimming behavior in the lamprey and leech, and swimmeret beating in the crayfish, are representative of more tightly coupled oscillators; arising as an emergent property of the locomotor CPGs (reviewed by: Skinner and Mulloney, 1998; Hill et al., 2003). Locomotor behaviors that require more adaptability, such as crawling or walking (Juvin et al., 2005; Borgmann et al., 2009), may preclude the configuration of oscillators that are tightly coupled and whose coordination is predominantly "built in" to the pattern-generating elements. 


\section{Interaction of local and long-distance signals}

One computational model of lamprey swimming (Cohen and Kiemel, 1993) suggests that interoscillator coupling can be achieved when stronger, shorter-distance, interoscillator signals are combined with long-distance projecting signals. This model can generate predictable interoscillator phase delays and without the need for precisely timed information stemming from putative long-distance projecting signals. If the interoscillator signals are stronger in the posterior direction, propagation will travel only in that direction, as we see in leech crawling. Our study also substantiated the role of a descending long-distance projecting signal.

Similarly, a model specifically for leech crawling predicted the need for a cephalic signal that projects through the length of the CNS (Cacciatore et al., 2000). Also proposed was the posteriordirected excitatory drive, between segments, as substantiated here (Figs. 7, 8,10). However, several important predictions of this model were not confirmed here. For example, Cacciatore et al. (2000) proposed that only a subset of ganglia contain the rhythm-generating elements for crawling. As we have shown here and in our previous report (Puhl and Mesce, 2008), a complete crawl pattern-generating circuit exists within every segmental ganglion.

These DA-biased crawl oscillators were found to be most stable either in complete isolation (Fig. 6) or when cephalic descending inputs were present (Fig. 4). Dopamine-treated collections of ganglia lacking cephalic inputs exhibited degraded crawl-like activity, supporting the idea that conflicting or inappropriate intersegmental signaling emerges when cephalic influences are absent.

Although we did not specifically identify the cephalic neurons that activate crawling and coordinate the intersegmental phase delays, several candidates could fulfill such functions. Most notable are the R3b cephalic projection neurons. In particular, cells R3b-1 and R3b-3 can activate or reset the crawl rhythm, and they show rhythmic bursting that is phase locked with spontaneous overt crawling in nearly intact leeches (Esch et al., 2002; Mesce et al., 2008). Both pairs have been shown to project uninterrupted through the nerve cord. Thus, the long-distance-projecting neurons incorporated into the model of Cohen and Kiemel (1993) do have a biological correlate in the leech, and warrant further study in terms of intersegmental coordination.

\section{Crawling, swimming, and their modification}

The necessity of cephalic input for crawling sharply contrasts with swimming, the other major form of locomotion in the leech. In the nearly intact animal, swimming was expressed spontaneously (Fig. $3 A-C$ ) in the absence of cephalic information. In fact, individual episodes of swimming were extended in the absence of descending input (Fig. 3C). Using the reversible sucrose block, our data reconfirm previous conclusions that the cephalic ganglion plays a largely inhibitory role over the decision to swim (Brodfuehrer and Friesen, 1986a; Brodfuehrer and Burns, 1995; Cornford et al., 2006). Once activated, the dynamics of swimming are fairly stereotypical and can continue with strong intersegmental coordination independent of cephalic input (Kristan and Calabrese, 1976).

Although the leech does possess gating cells for swimming, they are distributed among the segmental ganglia housing the swim oscillators. The most prominent of these swim gating cells is cell 204, which projects through Faivre's nerve (Weeks and Kristan, 1978). By severing this nerve, we showed that cell 204 is not needed to gate crawling (Fig. 9A). The segmental distribution of cell 204, in combination with a series of tightly coupled oscil- lators, may help to explain why swimming is less dependent on higher-order descending control. Because the swim circuitry has already been studied in exquisite detail (for review, see: Brodfuehrer et al., 1995; Kristan et al., 2005), future studies of crawling may reveal functional architectures optimized for variable (slow) versus stereotypic (fast) forms of locomotion, features likely conserved across animal groups.

Unlike the "trigger" neurons for swimming (Brodfuehrer and Friesen, 1986b), crawl-activating cells in the cephalic ganglion express gating properties; their activity must be sustained for crawl cycles to be repeated (Esch et al., 2002). In addition to providing the coordinating signal, on-going cephalic activity might also route sensory and efferent signals vital for postural or reflexive adjustments during crawling (Mesce et al., 2008). Although we did not focus on the effects of sensory systems to entrain or reset the crawl oscillators, our preliminary data indicate that mechanosensory inputs can alter their timing (Puhl and Mesce, unpublished observations). In the stick insect, sensory signals from stepping front legs can entrain the on-going stepping of middle and back legs; however, local load signals in middle and hind legs can override this synchronization (Borgmann et al., 2009). Regardless of the signal source (i.e., sensory or cephalic signals), important parallels emerge: the intersegmental activation and coordination of segmental oscillators in tandem with local modification enable an animal to overlay an array of movements or searching-related behaviors while locomoting; all performed without missing a step.

\section{References}

Akay T, McVea DA, Tachibana A, Pearson KG (2006) Coordination of fore and hind leg stepping in cats on a transversely-split treadmill. Exp Brain Res 175:211-222.

Borgmann A, Hooper SL, Büschges A (2009) Sensory feedback induced by front-leg stepping entrains the activity of central pattern generators in caudal segments of the stick insect walking system. J Neurosci 29:2972-2983.

Brodfuehrer PD, Burns A (1995) Neuronal factors influencing the decision to swim in the medicinal leech. Neurobiol Learn Mem 63:192-199.

Brodfuehrer PD, Friesen WO (1986a) Control of leech swimming activity by the cephalic ganglia. J Neurobiol 17:697-705.

Brodfuehrer PD, Friesen WO (1986b) Initiation of swimming activity by trigger neurons in the leech subesophageal ganglion. I. Output connections of Tr1 and Tr2. J Comp Physiol A 159:489-502.

Brodfuehrer PD, Debski EA, O'Gara BA, Friesen WO (1995) Neuronal control of leech swimming. J Neurobiol 27:403-418.

Büschges A (2005) Sensory control and organization of neural networks mediating coordination of multisegmental organs for locomotion. J Neurophysiol 93:1127-1135.

Büschges A, Akay T, Gabriel JP, Schmidt J (2008) Organizing network action for locomotion: insights from studying insect walking. Brain Res Rev 57:162-171.

Cacciatore TW, Rozenshteyn R, Kristan WB Jr (2000) Kinematics and modeling of leech crawling: evidence for an oscillatory behavior produced by propagating waves of excitation. J Neurosci 20:1643-1655.

Cang J, Friesen WO (2002) Model for intersegmental coordination of leech swimming: central and sensory mechanisms. J Neurophysiol 87:2760-2769.

Cohen AH, Kiemel T (1993) Intersegmental coordination: lessons from modeling systems of coupled non-linear oscillators. Am Zool 33:54-65.

Cohen AH, Wallén P (1980) The neuronal correlate of locomotion in fish. "fictive swimming" induced in an in vitro preparation of the lamprey spinal cord. Exp Brain Res 41:11-18.

Cornford A, Kristan WB 3rd, Malnove S, Kristan WB Jr, French KA (2006) Functions of the subesophageal ganglion in the medicinal leech revealed by ablation of neuromeres in embryos. J Exp Biol 209:493-503.

Crisp KM, Mesce KA (2004) A cephalic projection neuron involved in locomotion is dye coupled to the dopaminergic neural network in the medicinal leech. J Exp Biol 207:4535-4542. 
Drew T, Rossignol S (1984) Phase-dependent responses evoked in limb muscles by stimulation of medullary reticular formation during locomotion in thalamic cats. J Neurophysiol 52:653-675.

Drew T, Dubuc R, Rossignol S (1986) Discharge patterns of reticulospinal and other reticular neurons in chronic, unrestrained cats walking on a treadmill. J Neurophysiol 55:375-401.

Eisenhart FJ, Cacciatore TW, Kristan WB Jr (2000) A central pattern generator underlies crawling in the medicinal leech. J Comp Physiol A 186: 631-643.

Esch T, Mesce KA, Kristan WB (2002) Evidence for sequential decision making in the medicinal leech. J Neurosci 22:11045-11054.

Friesen WO, Brodfuehrer PD (1984) Identification of neurones in the leech through local ionic manipulations. J Exp Biol 113:455-460.

Garcia-Rill E, Skinner RD (1987) The mesencephalic locomotor region. I. Activation of a medullary projection site. Brain Res 411:1-12.

Georgopoulos AP, Grillner S (1989) Visuomotor coordination in reaching and locomotion. Science 245:1209-1210.

Gilchrist LS, Klukas KA, Jellies J, Rapus J, Eckert M, Mesce KA (1995) Distribution and developmental expression of octopamine-immunoreactive neurons in the central nervous system of the leech. J Comp Neurol 353:451-463

Gordon IT, Whelan PJ (2008) Brainstem modulation of locomotion in the neonatal mouse spinal cord. J Physiol 586:2487-2497.

Gray J, Lissman HW, Pumphrey RJ (1938) The mechanism of locomotion in the leech (Hirudo medicinalis). J Exp Biol 15:408-430.

Grillner S (2003) The motor infrastructure: from ion channels to neuronal networks. Nat Rev Neurosci 4:573-586.

Gruhn M, Zehl L, Büschges A (2009) Straight walking and turning on a slippery surface. J Exp Biol 212:194-209.

Hill AA, Masino MA, Calabrese RL (2003) Intersegmental coordination of rhythmic motor patterns. J Neurophysiol 90:531-538.

Ikeda K, Wiersma CA (1964) Autogenic rhythmicity in the abdominal ganglia of the crayfish: the control of swimmeret movements. Comp Biochem Physiol 12:107-115.

Ivanenko YP, Poppele RE, Lacquaniti F (2009) Distributed neural networks for controlling human locomotion: lessons from normal and SCI subjects. Brain Res Bull 78:13-21.

Juvin L, Simmers J, Morin D (2005) Propriospinal circuitry underlying interlimb coordination in mammalian quadrupedal locomotion. J Neurosci 25:6025-6035.

Kiehn O, Butt SJ (2003) Physiological, anatomical and genetic identification of CPG neurons in the developing mammalian spinal cord. Prog Neurobiol 70:347-361.

Kristan WB Jr, Calabrese RL (1976) Rhythmic swimming activity in neurones of the isolated nerve cord of the leech. J Exp Biol 65:643-668.

Kristan WB Jr, Stent GS, Ort CA (1974) Neuronal control of swimming in the medicinal leech. I. Dynamics of the swimming rhythm. J Comp Physiol A 94:97-119.

Kristan WB Jr, McGirr SJ, Simpson GV (1982) Behavioral and mechanosensory neurone responses to skin stimulation in leeches. J Exp Biol 96:143-160.

Kristan WB Jr, Calabrese RL, Friesen WO (2005) Neuronal control of leech behavior. Prog Neurobiol 76:279-327.

Lavoie S, McFadyen B, Drew T (1995) A kinematic and kinetic analysis of locomotion during voluntary gait modification in the cat. Exp Brain Res 106:39-56
Masino MA, Calabrese RL (2002) Period differences between segmental oscillators produce intersegmental phase differences in the leech heartbeat timing network. J Neurophysiol 87:1603-1615.

Mason A, Kristan WB Jr (1982) Neuronal excitation, inhibition and modulation of leech longitudinal muscle. J Comp Physiol A 146:527-536.

McClellan AD, Grillner S (1984) Activation of 'fictive swimming' by electrical microstimulation of brainstem locomotor regions in an in vitro preparation of the lamprey central nervous system. Brain Res 300:357-361.

Mesce KA, Esch T, Kristan WB Jr (2008) Cellular substrates of action selection: A cluster of higher-order descending neurons shapes body posture and locomotion. J Comp Physiol A 194:469-481.

Mori S, Nishimura H, Kurakami C, Yamamura T, Aoki M (1978) Controlled locomotion in the mesencephalic cat: distribution of facilitatory and inhibitory regions within pontine tegmentum. J Neurophysiol 41: $1580-1591$.

Nicholls JG, Baylor DA (1968) Specific modalities and receptive fields of sensory neurons in CNS of the leech. J Neurophysiol 31:740-756.

Pearce RA, Friesen WO (1985) Intersegmental coordination of the leech swimming rhythm. II. Comparison of long and short chains of ganglia. J Neurophysiol 54:1460-1472.

Puhl JG, Mesce KA (2008) Dopamine activates the motor pattern for crawling in the medicinal leech. J Neurosci 28:4192-4200.

R Development Core Team (2009) R: a language and environment for statistical computing. Vienna: R Foundation for Statistical Computing.

Robertson RM, Pearson KG (1983) Interneurons in the flight system of the locust: distribution, connections, and resetting properties. J Comp Neurol 215:33-50.

Shimamura M, Kogure I (1983) Discharge patterns of reticulospinal neurons corresponding with quadrupedal leg movements in thalamic cats. Brain Res 260:27-34.

Siddall ME, Trontelj P, Utevsky SY, Nkamany M, Macdonald KS (2007) Diverse molecular data demonstrate that commercially available medicinal leeches are not Hirudo medicinalis. Proc Biol Sci 274:1481-1487.

Skinner FK, Mulloney B (1998) Intersegmental coordination in invertebrates and vertebrates. Curr Opin Neurobiol 8:725-732.

Smarandache C, Hall WM, Mulloney B (2009) Coordination of rhythmic motor activity by gradients of synaptic strength in a neural circuit that couples modular neural oscillators. J Neurosci 29:9351-9360.

Stern-Tomlinson W, Nusbaum MP, Perez LE, Kristan WB Jr (1986) A kinematic study of crawling behavior in the leech, Hirudo medicinalis. J Comp Physiol A 158:593-603.

Stuart AE (1970) Physiological and morphological properties of motoneurones in the central nervous system of the leech. J Physiol 209:627-646.

Szczupak L, Edgar J, Peralta ML, Kristan WB Jr (1998) Long-lasting depolarization of leech neurons mediated by receptors with a nicotinic binding site. J Exp Biol 201:1895-1906.

Tschuluun N, Hall WM, Mulloney B (2001) Limb movements during locomotion: tests of a model of an intersegmental coordinating circuit. J Neurosci 21:7859-7869.

Weeks JC, Kristan WB Jr (1978) Initiation, maintenance and modulation of swimming in the medicinal leech by the activity of a single neuron. J Exp Biol 77:71-88.

Yang JF, Gorassini M (2006) Spinal and brain control of human walking: implications for retraining of walking. Neuroscientist 12:379-389. 